\title{
Optimization Framework for Conceptual Powertrain
}

\section{Design}

Tobias Hellberg and Martin Meywerk

Institute of Automotive and Powertrain Engineering, Helmut-Schmidt-University, Hamburg 22043, Germany

\begin{abstract}
With an increasing number of vehicles with alternative powertrains, the choice of the most appropriate powertrain system for a vehicle class or a load cycle is challenging. This paper introduces a method to design an optimal alternative powertrain based on a longitudinal dynamic simulation. The objective function of the minimization problem describes the characteristic map of the traction system. The goal of the optimization is to minimize fuel consumption respectively energy demand. Different types of propulsion systems are investigated. The results show that the proposed method delivers useful alternative powertrains by applying an optimization with reasonable restrictions.
\end{abstract}

Key words: Simulation in automotive engineering, hybrid powertrain, optimization, longitudinal dynamic vehicle simulation.

\section{Introduction}

The diversity of powertrain configurations in passenger cars has increased in recent years due to intensified search for resource efficiency. Main reason for the diversification is the electrification of the powertrain. Besides the ICE (internal combustion engine) as standard propulsion system, there are numerous configurations with an EM (electric machine) and ICE combined in a propulsion system (HEV (hybrid electric vehicle)) or an EM as standalone solution (BEV (battery electric vehicle)). Consequently, the identification of an optimal powertrain for a specific application or load spectrum has become a challenging task. This paper presents a method to obtain an optimal propulsion system on predefined load spectra using an optimization of vehicle specific parameters with a longitudinal dynamic simulation for different types of propulsion systems. The method is illustrated by example of the NEDC (New European Driving Cycle) and the Worldwide harmonized Light duty driving Test Cycle (WLTC).

Corresponding author: Tobias Hellberg, Dipl.-Ing., research fields: hybrid powertrains, optimization and test rig automation. E-mail: tobias.hellberg@hsu-hh.de.
An overview of different types of propulsion systems is given in Section 2; in Section 3, the considered load cycles are described, and the differences between the NEDC and WLTC and their impact on different propulsion systems are shown; the simulation model and mathematical description of a characteristic map are introduced in Sections 4 and 5. The formulation of the constrained nonlinear optimization problem as well as the optimization results is shown in Section 6; the conclusions of this paper are presented in Section 7.

\section{Types of Propulsion Systems}

Propulsion systems for passenger cars can be categorized into three main groups as shown in Fig. 1. The first group is the ICEs consisting of diesel and petrol engines, which are frequently combined with a turbocharger using the enthalpy within exhaust gases to get an efficiency improvement. This group makes up a share of approximately 99\% of all new car registrations in the USA, Japan, China and Germany [1]. The second group is BEVs with an electric machine as traction power engine. In passenger cars, 


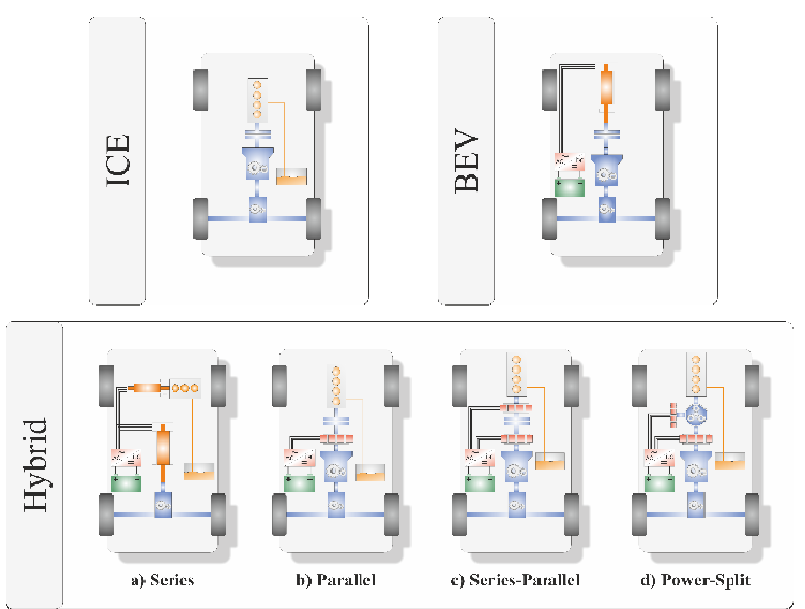

Fig. 1 Powertrain configurations.

mainly two types of electric machines are used: AIM (asynchronous induction machines) and PMSM (permanent magnet synchronous machines). AIM is lower-priced compared to the high costs of PMSM resulting from the use of rare earth metals. But AIM does not have the power density and high efficiency at low and moderate speeds as the PMSM. The third group of propulsion systems is hybrid powertrains with at least two engines that can be categorized into Series, Parallel, Series-Parallel and Power-Split Hybrid powertrains [2]. Fig. 1 shows exemplary structures of hybrid powertrains out of a vast number of possible configurations [3].

The series hybrid (Fig. 1a) is characterized by the fact that the traction energy is delivered by the electric machine. To produce electrical energy, an ICE drives a generator. The electrical energy is saved in a battery or is directly used by the traction EM. There are two principle design variants. If the vehicle should permanently deliver the same driving performance, all engines have to be designed for maximum velocity [4], this leads to high costs. Alternatively the series hybrid is designed as shown in Fig. 1a. The ICE and the generator are both smaller, so that the ICE can operate at higher efficiency and is lower in costs. The disadvantage is that the driving performance will decrease when the SOC (state of charge) of the battery is low.

A possible parallel hybrid design is shown in Fig. 1b. The traction energy can be provided by either the ICE, the EM or both of them. Several locations of the EM and the clutch are possible. The power of the electric machine is another design parameter. Micro-Hybrids have an installed electrical power of max. $6 \mathrm{~kW}$. In these hybrids, the electrical machine has only supportive function of the ICE, consequently, zero emission driving is merely possible in a very limited load and speed range. Full hybrids have a greater capacity of electric power $(>40 \mathrm{~kW})$, so that nearly all driving tasks can be solved with the EM. This kind of hybrid enables local zero emission driving. Plug-in hybrids are characterized by a battery that is chargeable from the grid. Depending on the capacity of the installed battery, electrical driving is possible up to $50 \mathrm{~km}$.

The series-parallel configuration (Fig. 1c) gives the possibility of charging the battery while the ICE and the EM are connected to the driving wheels. The load point of the ICE can be shifted to increase efficiency. In this operation mode, the generator gives an additional load on the ICE and charges the battery. It is also possible to operate both electric machines combined for a boost mode to get higher accelerations.

Fig. 1d shows a variation of a power-split hybrid. Contrary to the series-parallel hybrid, the generator cannot support the traction machines, but the planetary gear makes a free power distribution between ICE and both of the electric machines possible.

\section{Load Spectra}

Load spectra can be interpreted as load collectives or load cycles and describe the usage of a vehicle related to the powertrain. It can be derived by analyzing the speed profile and the topology for the travelled route. The power and energy demand can be obtained analyzing vehicle data and are unique for a specific use case. In this paper two standardized cycles are used to ensure comparability of energy demand respectively fuel consumption of different 
vehicles. The NEDC is used in European countries to determine the energy and fuel consumption. The worldwide harmonized light vehicle test cycle (WTLC) will replace the NEDC in the near future. The WLTC is part of the worldwide harmonized light vehicle test procedure (WTLP). These two cycles will be used to test the designed method. The characteristics of these cycles will be described in the next section. In this paper the cycles are only used to get a standardized load cycle and to test the designed method. Therefore some of the regulations of those cycles will be neglected, e.g., calculating the weighted average of fuel consumption for hybrid vehicles.

Both cycles, NEDC and WLTC, are chosen to test the proposed method for the design of an optimal propulsion system because the requirements of the two cycles are different. The design of the speed profiles of the NEDC shows a focus that differs from the WLTC (Fig. 2). The dashed lines show the separate sections of the cycles. Within the NEDC the urban cycle is passed four times and has a duration of $780 \mathrm{~s}$ in total. The extra urban cycle has a duration of $400 \mathrm{~s}$ and shows higher speeds to represent country road and highway driving.

The WTLC is divided into four sections. They are named low, mid, high and extra high according to the speeds driven in the section. The NEDC consists of a sequence of straight lines in the speed-over-time graph. Every sequence of the NEDC has a constant acceleration and a mean duration of $7.8 \mathrm{~s}$ for the urban cycle and $19 \mathrm{~s}$ for the extra-urban cycle. Contrary to that, the acceleration is not constant when the vehicle is driving in WLTC.

Because of the persistent criticism that the NEDC delivers too low fuel consumption compared to the actually observed fuel consumption of the customer, the overall energy demand in the WLTC has been set higher than in the NEDC. Additionally, the covered distance in the WLTC is more than twice as long as in the NEDC. For a C-segment or compact car the total
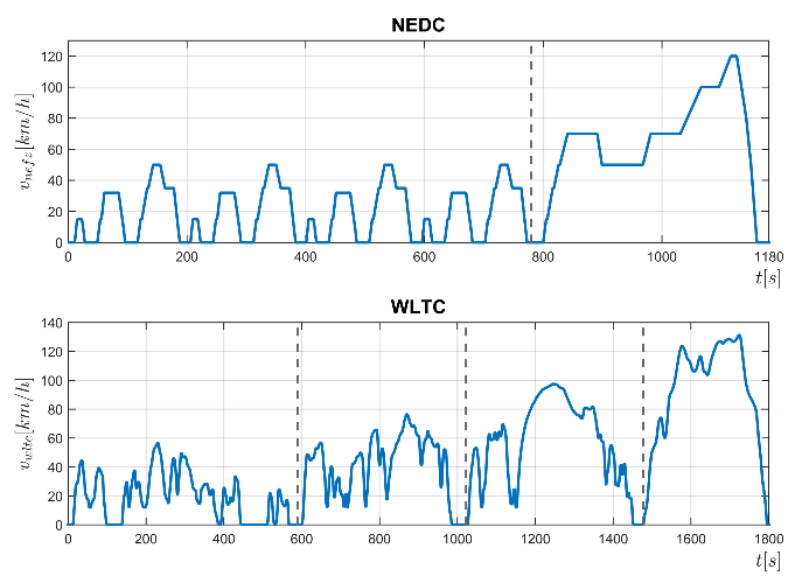

Fig. 2 Speed over time profile of the NEDC and WTLC.

energy demand for the WLTC is about $E_{\text {tot,WLTC }}=13$ MJ with a covered distance of $s_{\text {tot,WLTC }}=23.3 \mathrm{~km}$. The total energy demand for the NEDC is about $E_{\text {tot,NEDC }}=$ $6.8 \mathrm{MJ}$ with a covered distance of $s_{\mathrm{tot}, \mathrm{NEDC}}=11 \mathrm{~km}$.

The higher loads lead to higher efficiency in a limited scope for an ICE or EM. Due to the fact that an EM in combination with an inverter is widely operating in a range of over $70 \%$ efficiency, the relative gain in efficiency for the EM is lower than that for the ICE.

\section{Simulation Model}

The longitudinal dynamic simulation (Fig. 3) is designed as a forward simulation. The load of the traction machines is not calculated from the road load backwards to the engine load (s) as in backward simulation, but from a driver model that follows a speed trajectory. The advantage of this approach is, that different types of drivers can be specified in further studies, and the behavior of the driver can serve as an additional influence factor for the optimization. The simulation model is validated with the support of coast-down tests. The coefficients of the objective function (thus the driving resistances polynomial) are determined through a least square optimization.

Three main systems interact in the simulation: the driver system, the vehicle system and the environment system. The driver system contains two control 


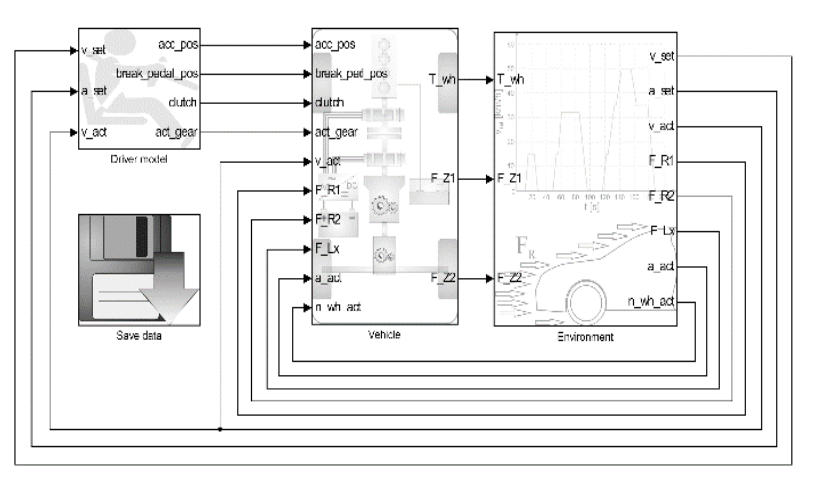

Fig. 3 Longitudinal simulation model.

structures: one for acceleration and one for deceleration (braking). The system switches between these two control structures using thresholds and conditions. In both states a PID controller is used to approximate the speed error to zero.

The vehicle system consists of the brake system, the propulsion systems, the gearbox and the operation strategy for hybrid vehicles. As a result, the wheel torque is transmitted to the environment system where tyre-road contact is taken into account and driving resistances are calculated. The acceleration of the vehicle is obtained by solving the equation of motion.

As variable gear ratios are needed for the optimization an ideally progressive design with fixed coefficients is used for the choice of the gear ratios. This reduces the number of free parameters for the optimization to two. One is for the final drive ratio and one for the highest gear from which the other gear ratios can be calculated.

The maps for fuel consumption and efficiency of the traction machines are stored in the vehicle system. Thus the maps can be varied during optimization runs. The time-depending output of the maps is saved and used as optimization target. The approach of how the maps are deducted will be discussed in the next section.

\section{Modeling Characteristic Maps}

The mathematical description of a characteristic specific fuel consumption map for an ICE and an efficiency map for an EM is a challenging task. To find an optimal propulsion system, it is indispensable to scale and shift the maps in a way that the majority of load points will be located in the region of minimum fuel consumption (maximum efficiency). This requires a simple mathematical description for the substitute of the maps with a small number of parameters to reduce complexity of the optimization problem. For this method a quadric surface in $\mathbb{R}^{3}$ is chosen, which is represented by the general formula

$$
\begin{aligned}
Q(\underline{x}) & =\sum_{i, j=1}^{3} a_{i j} x_{i} x_{j}+\sum_{i=1}^{3} b_{i} x_{i}+c \\
& =\underline{\mathrm{x}}^{T} \underline{\underline{\mathrm{A}}} \underline{\mathrm{x}}+\mathrm{b}^{T} \underline{\mathrm{x}}+\mathrm{c}=0
\end{aligned}
$$

The vector $\underline{\mathrm{x}}=\left(x_{1}, x_{2}, x_{3}\right)^{T} \quad$ describes the variables, the vector $\underline{\mathrm{b}}$ contains the coefficients for the linear term, the matrix $\mathrm{A}$ is real and symmetric and contains the coefficients for the quadratic terms. As there are many different forms of three-dimensional hypersurfaces, an elliptic paraboloid turns out to meet the requirements for modelling characteristic maps best. The normal form of this quadric is

$$
\frac{x_{1}^{2}}{\rho_{1}^{2}}+\frac{x_{2}^{2}}{\rho_{2}^{2}}-\frac{x_{3}}{\rho_{3}}=0
$$

For this specific use, the three variables $x_{i}$ are the rotational speed, the output torque and the specific fuel consumption (efficiency) of the engine. The equation of the second-order surface is extended to get a higher degree of freedom. Therefore, it is possible to shift and rotate the paraboloid about every direction of the parameter space. The related equation in matrix notation is

$$
\tilde{Q}(\underline{\mathrm{x}})=\underline{\tilde{x}}^{T} \underline{\underline{\mathrm{A}}} \underline{\tilde{\mathrm{x}}}+\underline{\mathrm{b}}^{T} \underline{\tilde{\mathrm{x}}}=0
$$

With

$$
\underline{\tilde{x}}=\underline{\underline{\mathrm{D}}}_{x_{3}} \underline{\underline{\mathrm{D}}}_{x_{2}} \underline{\underline{\mathrm{D}}}_{x_{1}}(\underline{\mathrm{x}}+\underline{\mathrm{d}})
$$

$D_{x_{i}}$ denotes rotation matrices and the vector $\underline{\mathrm{d}}$ describes the translation. Thus Eq. (3) has nine free parameters (three angles of rotation, three translation 
parameters and three scaling parameters $\rho_{1}, \rho_{2}, \rho_{3}$ ) which can be varied in an optimization algorithm to fit this quadric to a specific real map. The least-square problem has the form

$$
\min _{x}\|q(\underline{\mathrm{x}})\|_{2}^{2}=\min _{x}\|\tilde{Q}(\underline{\mathrm{x}})-M(\underline{\mathrm{x}})\|_{2}^{2}
$$

$M(x)$ is the real characteristic map. Solving this problem under the constraint that the solution is always in the range $\underline{\mathrm{x}}_{l b} \leq \underline{\mathrm{x}} \leq \underline{\mathrm{x}}_{u b}$ (where $\underline{\mathrm{x}}_{l b}$ and $\underline{\mathrm{x}}_{u b}$ are the lower and upper boundary vectors), the obtained hypersurface is a representative for the real characteristic map. The minimization problem is formulated over a limited area of rotational speed to improve accuracy of the results for the ICE, because during the considered cycles the rotational speed of the engine will not exceed $50 \%$ of the maximum engine speed. Fig. 4 shows two fittings of the paraboloid.

In Fig. 4a, the surface is fitted over a characteristic map of a downsized, turbocharged petrol engine. The second-order surface has a small relative deviation to the ICE map $\mathrm{M}(\mathrm{x})$ at the relevant partial load for a wide speed range. The same applies to the characteristic map of a permanent magnet synchronous machine in Fig. 4b.

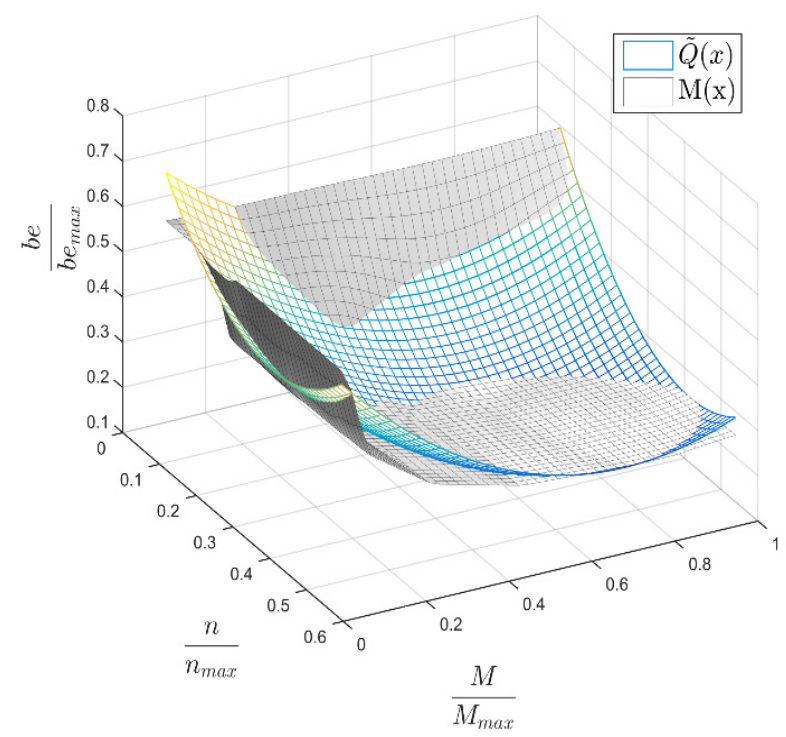

(a)
The results show a good correlation with manufacturer's specification for the fuel and power consumption using the simulation model with the presented surfaces for a test over the NEDC for a compact car. The relative deviation in combined power consumption for a $\mathrm{BEV}$ is $\Delta E_{\text {com }}=3.2 \%$, for the petrol engine the combined relative fuel consumption deviation is $\Delta B_{\text {com }}=1.3 \%$. These small differences in the consumption show that the elliptic paraboloid is suitable to approximate characteristic maps of internal combustion engines as well as electrical machines. In consequence this type of hypersurface is also able to represent hybrid electric vehicles, as their propulsion systems are merely a combination of an ICE and an EM.

\section{Definition of the Optimization}

The definition of the elliptic paraboloid as stated in (3) has nine free parameters in a nonlinear multivariable function. A gradient method is chosen for the optimization because the function is continuously differentiable. A SQP (sequential quadratic programming) method is used for this constrained nonlinear programming problem of the form

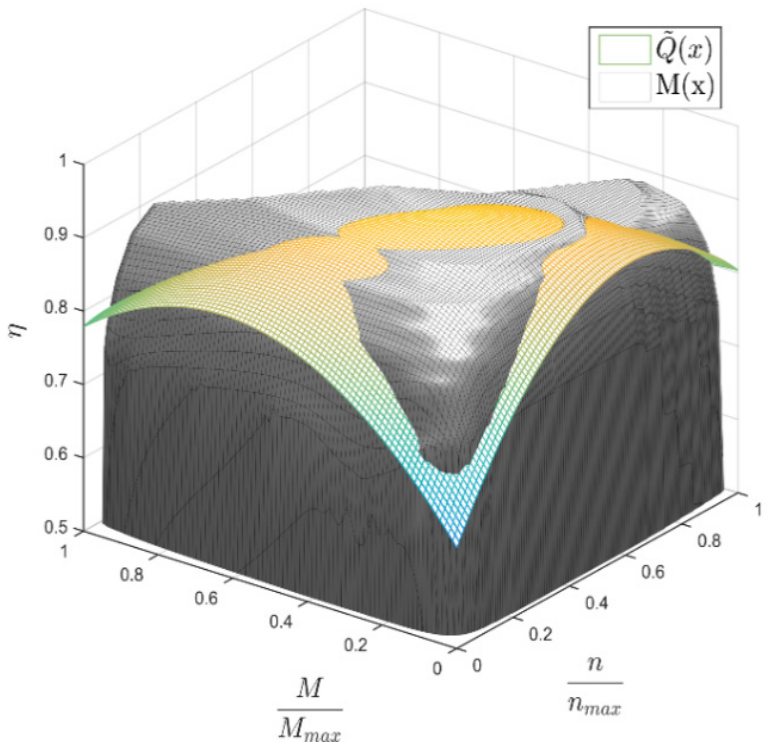

(b)

Fig. 4 Least-square problem results for an ICE and an EM characteristic map. 


$$
\begin{aligned}
B(\underline{\mathrm{x}}) & =\min ! \\
\text { over } \quad \underline{\mathrm{x}} & \in \mathbb{R}^{n} \\
\text { subject to } \underline{\mathrm{x}}_{l b} & \leq \underline{\mathrm{x}} \leq \underline{\mathrm{x}}_{u b} \\
\underline{\mathrm{H}} \underline{\mathrm{x}} & =\mathrm{g}
\end{aligned}
$$

$\mathrm{B}$ is the fuel consumption for ICE driven vehicles and the power consumption for BEV. The boundary condition vectors $\underline{x}_{b b}$ and $\underline{x}_{u b}$ define limits for the free parameters in Eq. (3). This ensures that the optimized elliptic paraboloid still has a shape that represents a real characteristic map. The equality condition in the stated optimization problem ensures that the minimum specific fuel consumption respectively the maximum efficiency have reasonable values.

The structure of the optimization problem with boundary conditions excludes Newton or Quasi-Newton method for solving the problem $[5,6]$. The SQP-Algorithm used for solving the stated problem is based on the algorithm of Nocedal and Wright [7]. The number of free parameters varies depending on the type of propulsion system used for the optimization. A single engine optimization has nine free parameters and a system with two engines has 18 free parameters for the optimization. Taking free gear ratios into account the number of free parameters is at least increased by two. One is for the gear ratios of the gearbox and one for the final drive. Due to the fact that every optimization step requires a simulation run, which typically takes about $25 \mathrm{~s}$ for the NEDC and approximately $35 \mathrm{~s}$ for the WLTC, the computation time for an optimization run varies from one and a half hour up to three hours for hybrid vehicles on the WLTC.

The minimization problem has to take fuel and power consumption into account when a hybrid vehicle is considered. In this paper only parallel hybrid vehicles will be investigated. The fuel/power consumption is the output of the simulation model. With ongoing iterations, the characteristic maps of the engines are varied up to the point where the reduction of fuel and/or power consumption per iteration is less than a predefined boundary value.
Three propulsion systems for a compact car are analyzed for a first evaluation of the designed optimization method: a petrol engine as a representative of the ICE, a PMSM for the BEV and a PHEV with a petrol engine and a PMSM in combination. All system was examined on NEDC and WLTC. The results will be presented in the next section.

\section{Results and Discussion}

The results for a PHEV are shown in Fig. 5. The torque over speed diagrams shows two different states for the traction engines. All black colored graphs represent the initial state, and the red colored graphs describe the state after the optimization run. In addition the load points for the considered cycle are shown with blue points.
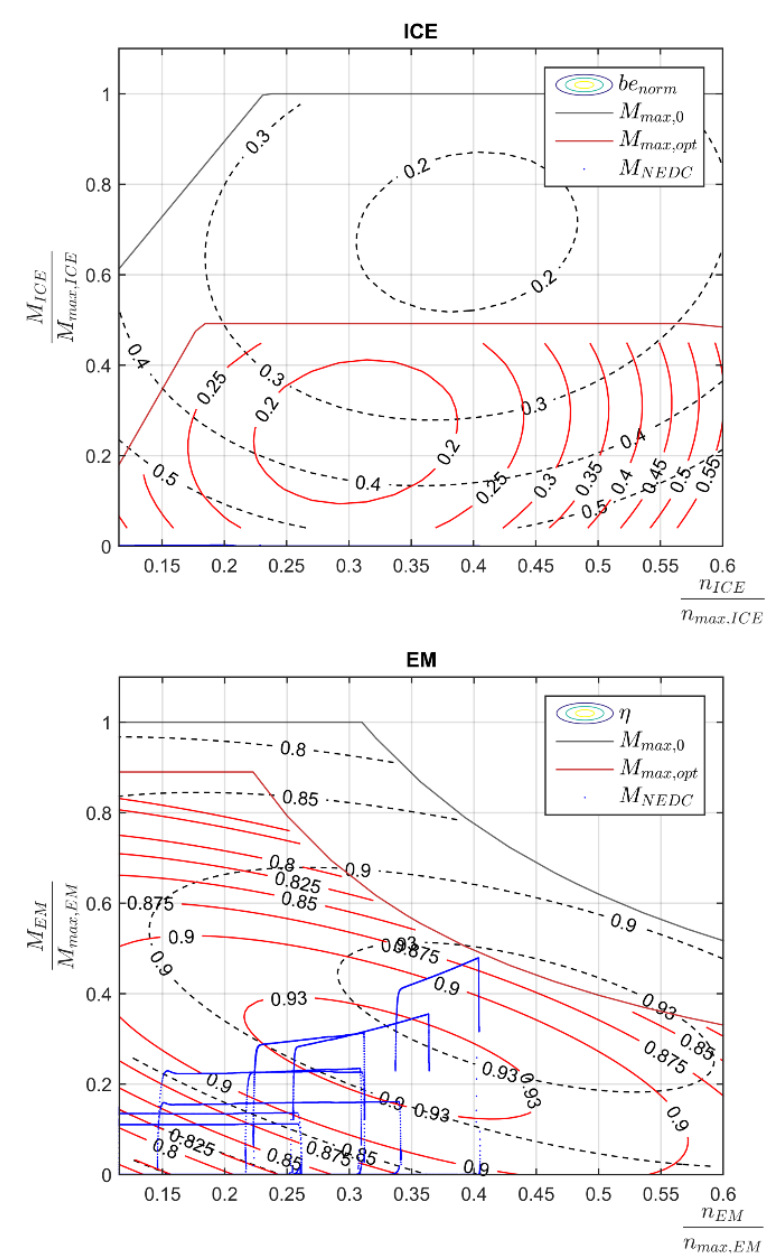

Fig. 5 Results for PHEV with charged battery on NEDC. 
The examined plug-in parallel hybrid vehicle with a charged battery is able to fulfill the whole cycle by using the EM only. As a result the efficiency map is shifted towards the emphasis of the load points until the boundary condition for the torque curve that all load points should be covered with the respective traction machine is met. The specific fuel consumption map of the ICE is shifted up to the limit of the minimum torque which is set to $50 \%$ of the initial value. An EM with approximately $48 \mathrm{~kW}$ peak power would be the optimal propulsion system for this use case of the considered vehicle. It has to be taken into account that the NEDC only covers a distance of approximately $11 \mathrm{~km}$. For a discharged battery the results are shown in Fig. 6.
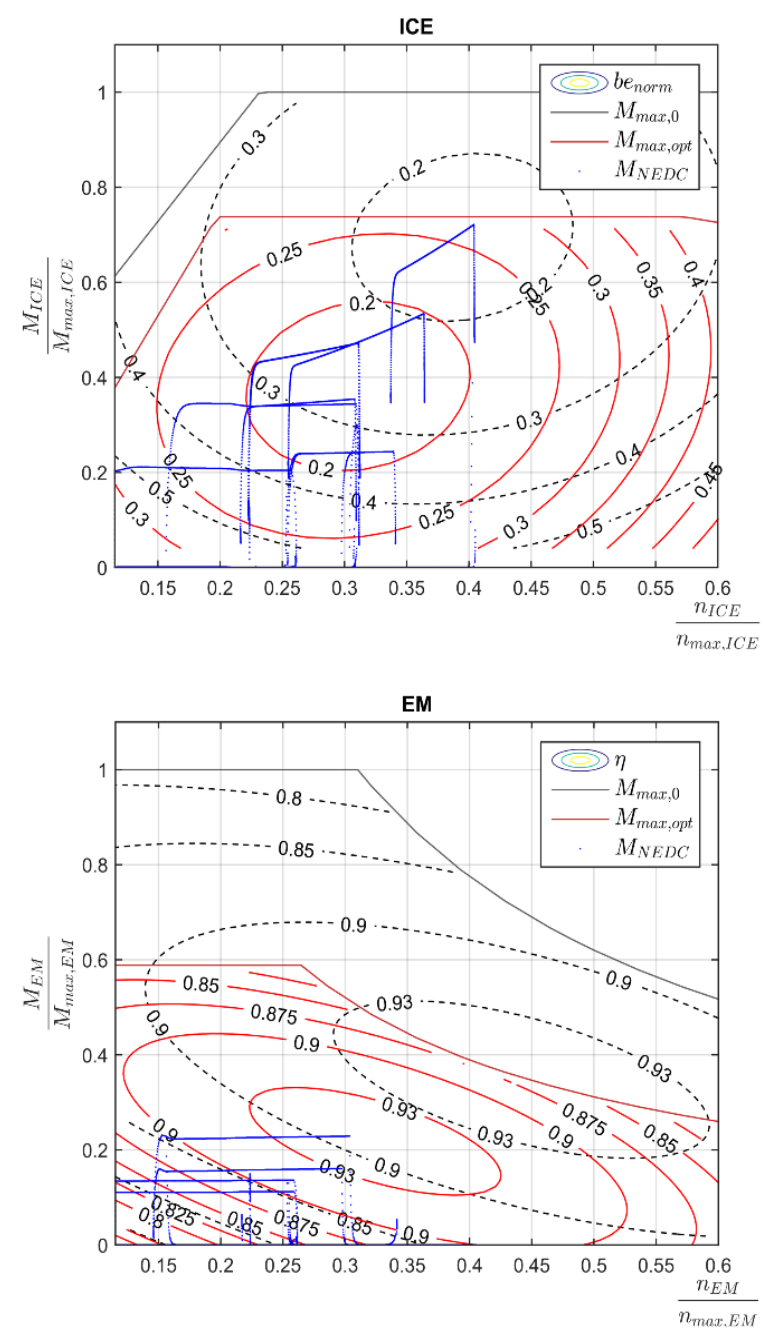

Fig. 6 Results for PHEV with discharged battery on NEDC.
With discharged battery at the beginning of the cycle, the optimization for the PHEV shows a different result. Only a limited load range can be covered with the EM because the amount of energy the battery of the PHEV is charged by recuperation during deceleration in urban cycle is small. The operational strategy for the PHEV does not allow load point shifting for charging the battery, thus recuperation is the only energy supplier for the battery. The efficiency map and the maximum torque curve are shifted down to lower values. Again a boundary condition is reached that the EM should be able to cover a speed range up to one hundred kilometers per hour. As the ICE is the main traction engine for this considered case the specific fuel consumption map is shifted as well as the efficiency map to lower values. In this case the optimization terminates because the decrease in fuel consumption per optimization step approaches zero.

In Fig. 7, the results for the petrol engine and the $\mathrm{BEV}$ are shown. Differences between the ICE driven vehicle compared to the ICE from the PHEV can be seen. Although both engines have nearly the same maximum torque, the optimization stops for the PHEV at a lower torque level. As the conventional vehicle is simulated with a manual 6-speed transmission and the PHEV with a 6-speed automatic transmission, the differences occur because of the coupling process. During shifting between the gears, the driver model reacts on an increasing speed error with a higher accelerator pedal position. This leads to a high torque demand and consequently to a termination of the optimization because of the boundary condition that every load point has to be reachable within the characteristic map.

The BEV has a single speed gearbox with a high ratio. This leads to significant lower torque and higher speed demands for the EM. The maximum rotational speed for the ICE is about $40 \%$ of the maximum speed. For the EM nearly $80 \%$ of the speed range is needed to cover the demand. The highest power is needed 

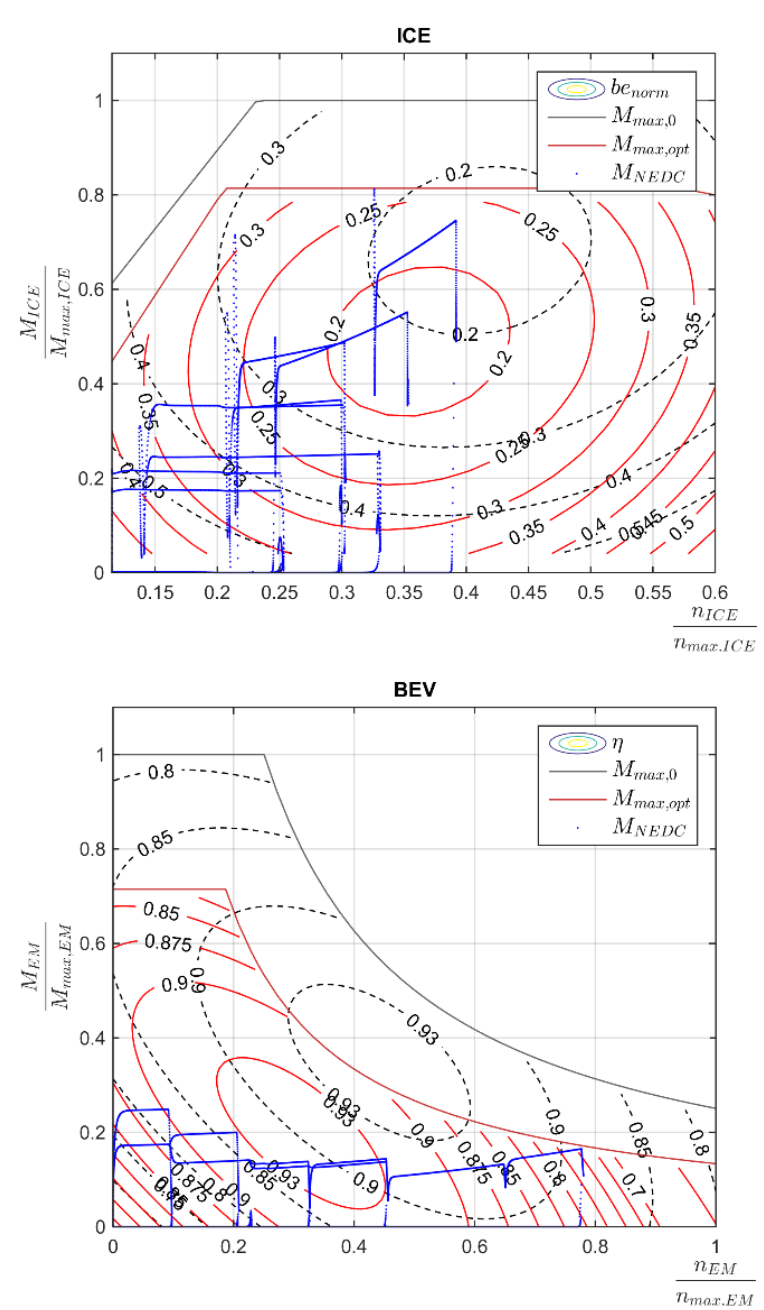

Fig. 7 Results for ICE and BEV on NEDC.

when accelerating to maximum speed. This is the point where the mentioned boundary condition is reached and the optimization terminates.

The optimization results for a PHEV with fully charged battery on the WLTC which is shown in Fig. 8. Because of the higher power demand for the WLTC, the optimization delivers a slightly different efficiency map respectively a slightly different torque curve for the charged PHEV with regard to the initial state.

Due to the fact that the required power to follow the speed trajectory in the extra-high section nearly reaches the power hyperbola in the field weakening region, there is no possibility to shift the map in a large scale. And the above mentioned boundary condition for the covered load points still holds for the WLTC. For the ICE the same result is achieved as for
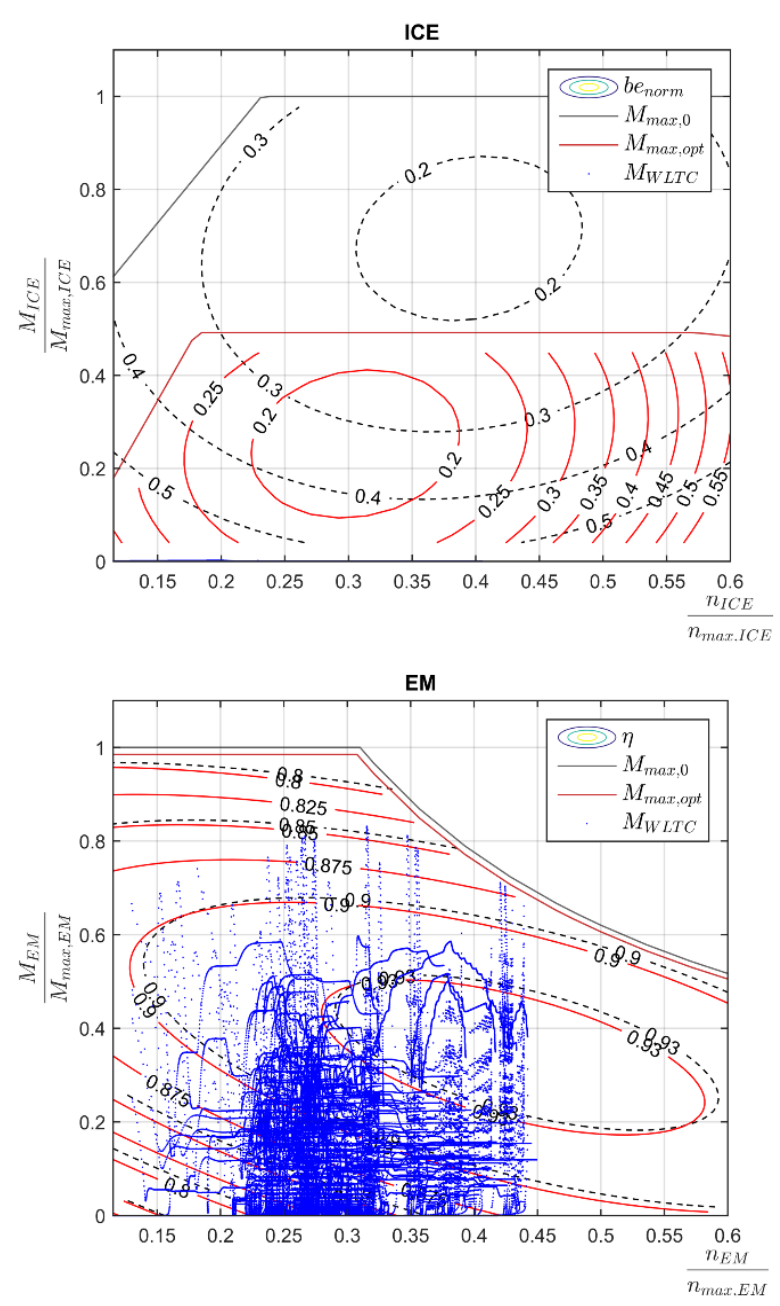

Fig. 8 Results for PHEV with charged battery on WLTC.

the charged PHEV on the NEDC. In contrast the results with discharged battery, shown in Fig. 9, differ from the results on the NEDC with the same configuration.

The optimization algorithm with its boundary conditions allows neither the specific fuel consumption map of the ICE nor the efficiency map of the EM to shift in a large scale. Due to the fact that both of them are nearly at full load at some points during the cycle, there is no possibility for shifting the maps in the direction of the emphasis of the load points.

The conventional vehicle and BEV on the WLTC show similar results to the PHEV (Fig. 10).

Because the torque demand for the ICE is in the range of full-load, the possibility to reduce the fuel 

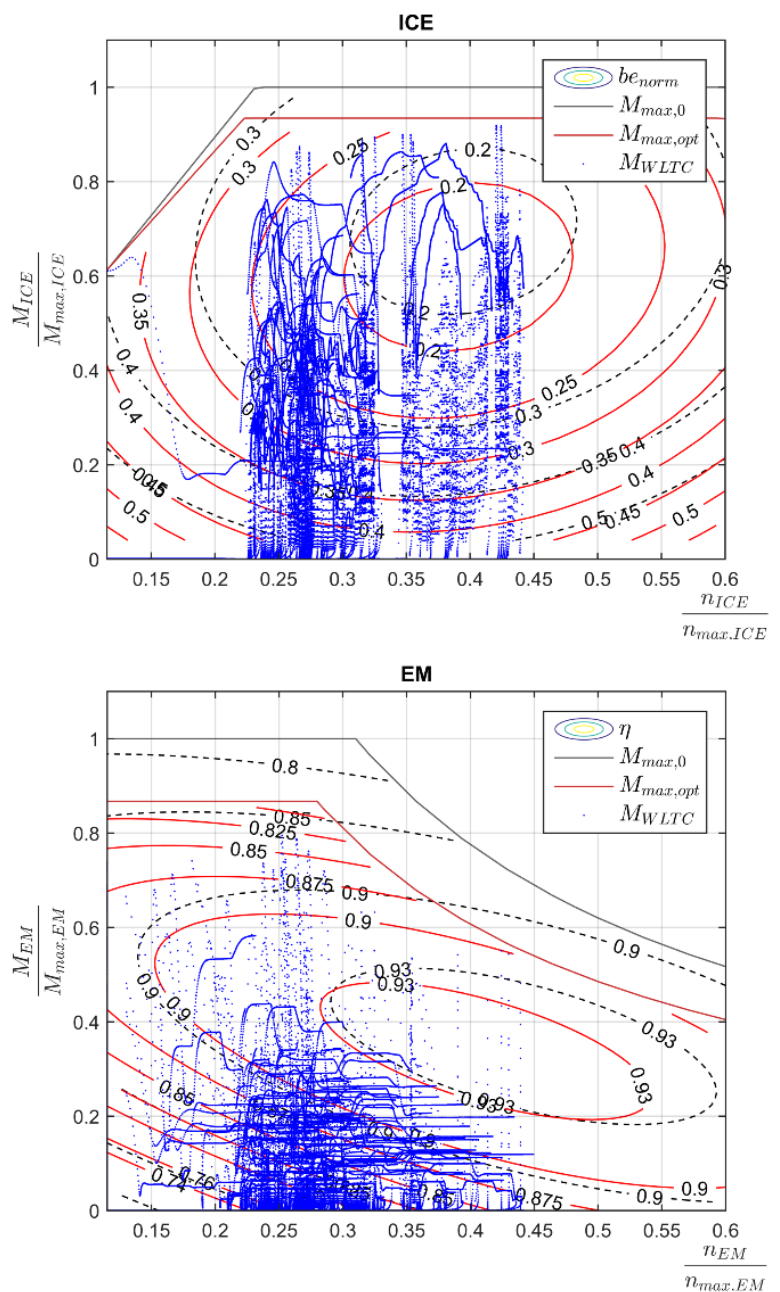

Fig. 9 Results for PHEV with discharged battery on WLTC.

consumption by shifting the characteristic maps is limited. The optimization possibilities are greater for the BEV with its high gearbox ratio. Although the torque demand at low speeds on the WLTC is nearly twice as high as on the NEDC, the demand only exceeds those of the NEDC in a small amount at high speed, which is also true for the power demand. Consequently, the efficiency map is shifted in a similar way.

To determine which propulsion system fits the considered load cycle best, a common basis for the analysis is needed. This basis can be found in the $\mathrm{CO}_{2}$ emissions of the traction system. For ICEs the relationship between fuel consumption and $\mathrm{CO}_{2}$ emissions is obvious. As EM has no local emissions,
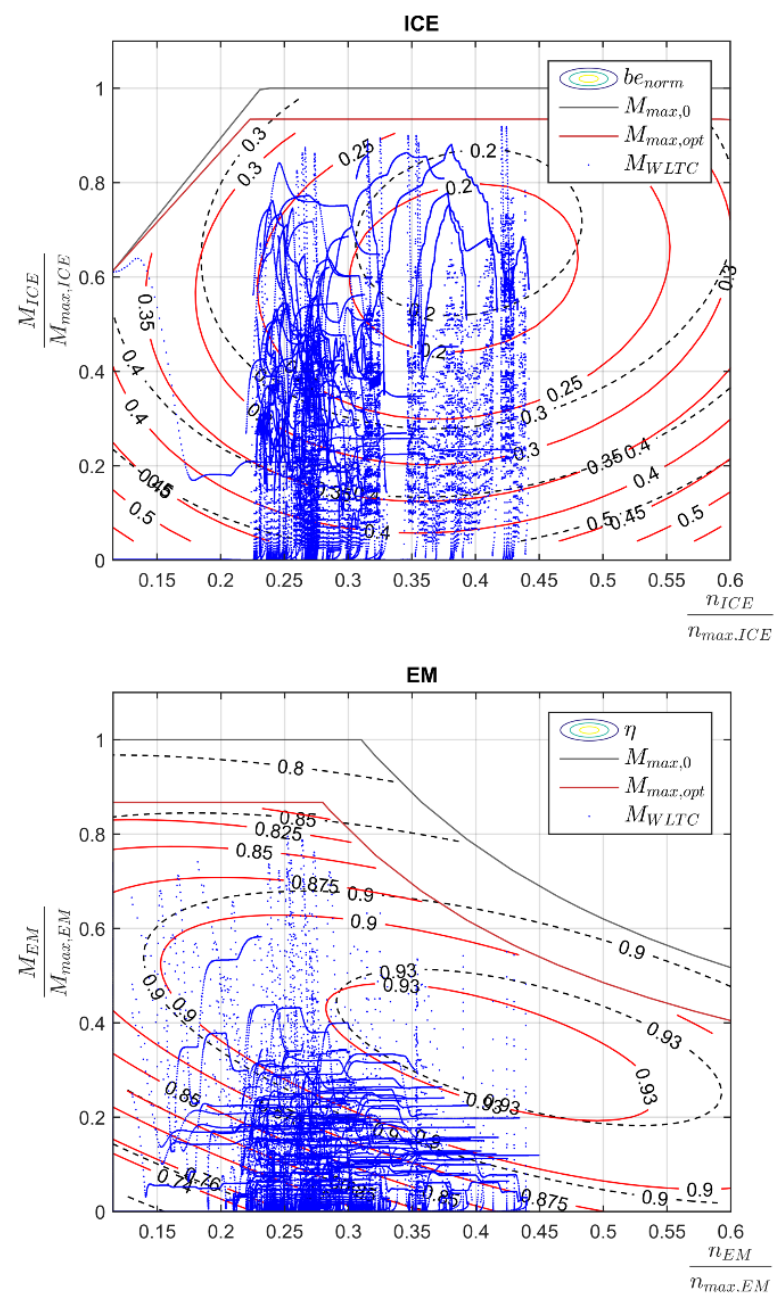

Fig. 10 Results for ICE and BEV on WLTC.

the emissions for the electricity generation have to be considered. In this paper, the $\mathrm{CO}_{2}$ emissions for the electricity generation in Germany's electricity mix are used to calculate the $\mathrm{CO}_{2}$ emissions for EM. For 2012, the $\mathrm{CO}_{2}$ emissions in Germany have been $562 \mathrm{~g} / \mathrm{kWh}$ [8]. Tables 1-4 show the results for the NEDC.

Table 1 Fuel consumption and $\mathrm{CO}_{2}$ emissions for conventional vehicle on the NEDC.

\begin{tabular}{lll}
\hline Conventional vehicle & $\mathrm{B}(1 / 100 \mathrm{~km})$ & $\mathrm{CO}_{2}(\mathrm{~g} / \mathrm{km})$ \\
\hline Initial & 5.3 & 122 \\
Optimized & 4.2 & 97 \\
\hline
\end{tabular}

Table 2 Energy consumption and $\mathrm{CO}_{2}$ emissions for $\mathrm{BEV}$ on the NEDC.

\begin{tabular}{lll}
\hline $\mathrm{BEV}$ & $\mathrm{E}(\mathrm{kWh} / 100 \mathrm{~km})$ & $\mathrm{CO}_{2}(\mathrm{~g} / \mathrm{km})$ \\
\hline Initial & 12.2 & 69 \\
Optimized & 11.4 & 64 \\
\hline
\end{tabular}


Table 3 Fuel/energy consumption and $\mathrm{CO}_{2}$ emissions for charged PHEV on the NEDC.

\begin{tabular}{llll}
\hline PHEV & $\mathrm{B}(\mathrm{l} / 100 \mathrm{~km})$ & $\mathrm{E}(\mathrm{kWh} / 100 \mathrm{~km})$ & $\mathrm{CO}_{2}(\mathrm{~g} / \mathrm{km})$ \\
\hline Initial & 0 & 14.0 & 79 \\
Optimized & 0 & 13.6 & 76 \\
\hline
\end{tabular}

Table 4 Fuel/Energy consumption and $\mathrm{CO}_{2}$ emissions for discharged PHEV on the NEDC.

\begin{tabular}{llll}
\hline PHEV & $\mathrm{B}(1 / 100 \mathrm{~km})$ & $\mathrm{E}(\mathrm{kWh} / 100 \mathrm{~km})$ & $\mathrm{CO}_{2}(\mathrm{~g} / \mathrm{km})$ \\
\hline Initial & 4.5 & 0 & 105 \\
Optimized & 3.2 & 0 & 78 \\
\hline
\end{tabular}

The BEV is the most suitable propulsion system for the NEDC based on the criteria used for this investigation. With an overall $\mathrm{CO}_{2}$-emission of $64 \mathrm{~g} / \mathrm{km}$ after the optimization, the BEV delivers the lowest value for this cycle. The charged PHEV emits the second lowest quantity of $\mathrm{CO}_{2}$. The ICE is not used due to the fact that the capacity of the battery is high enough to cover the energy demand in this cycle. In contrast, the discharged PHEV has to start with the ICE only and the battery is charged through the recuperation during deceleration phases. After deceleration, the EM can support the ICE, which leads to lower fuel consumption compared to the conventional vehicle.

For the WLTC a similar result is obtained (Tables 5-8). The ranking of the propulsion systems is the same, but the $\mathrm{CO}_{2}$-emission level is higher. The initial goals have been reached. A lower fuel/energy consumption has been achieved for every investigated propulsion system. A propulsion system fitted on the used load cycle increases efficiency and decreases $\mathrm{CO}_{2}$-emissions. As the underlying optimization algorithm is a gradient method, it cannot be ruled out that a local minimum has been found.

A further investigation was made on changing ratios of the transmission. In a first step, only the PHEV on the NEDC was evaluated. Fig. 11 shows the result for the PHEV with depleted battery.

The changing in the transmission ratios to higher values leads to a shift of the load points to higher engine speeds. Thus the load points are lying at higher engine efficiency and therefore the energy demand
Table 5 Fuel consumption and $\mathrm{CO}_{2}$ emissions for conventional vehicle on the WLTC.

\begin{tabular}{lll}
\hline Conventional vehicle & $\mathrm{B}(1 / 100 \mathrm{~km})$ & $\mathrm{CO}_{2}(\mathrm{~g} / \mathrm{km})$ \\
\hline Initial & 6.2 & 143 \\
Optimized & 5.7 & 131 \\
\hline
\end{tabular}

Table 6 Energy consumption and $\mathrm{CO}_{2}$ emissions for $\mathrm{BEV}$ on the WLTC.

\begin{tabular}{lll}
\hline $\mathrm{BEV}$ & $\mathrm{E}(\mathrm{kWh} / 100 \mathrm{~km})$ & $\mathrm{CO}_{2}(\mathrm{~g} / \mathrm{km})$ \\
\hline Initial & 14.4 & 81 \\
Optimized & 13.3 & 75 \\
\hline
\end{tabular}

Table 7 Fuel/Energy consumption and $\mathrm{CO}_{2}$ emissions for charged PHEV on the WLTC.

\begin{tabular}{llll}
\hline PHEV & $\mathrm{B}(1 / 100 \mathrm{~km})$ & $\mathrm{E}(\mathrm{kWh} / 100 \mathrm{~km})$ & $\mathrm{CO}_{2}(\mathrm{~g} / \mathrm{km})$ \\
\hline Initial & 0 & 16.2 & 91 \\
Optimized & 0 & 15.9 & 89 \\
\hline
\end{tabular}

Table 8 Fuel/Energy consumption and $\mathrm{CO}_{2}$ emissions for discharged PHEV on the WLTC.

\begin{tabular}{llll}
\hline PHEV & $\mathrm{B}(1 / 100 \mathrm{~km})$ & $\mathrm{E}(\mathrm{kWh} / 100 \mathrm{~km})$ & $\mathrm{CO}_{2}(\mathrm{~g} / \mathrm{km})$ \\
\hline Initial & 5.5 & 0 & 127 \\
Optimized & 4.5 & 0 & 104 \\
\hline
\end{tabular}
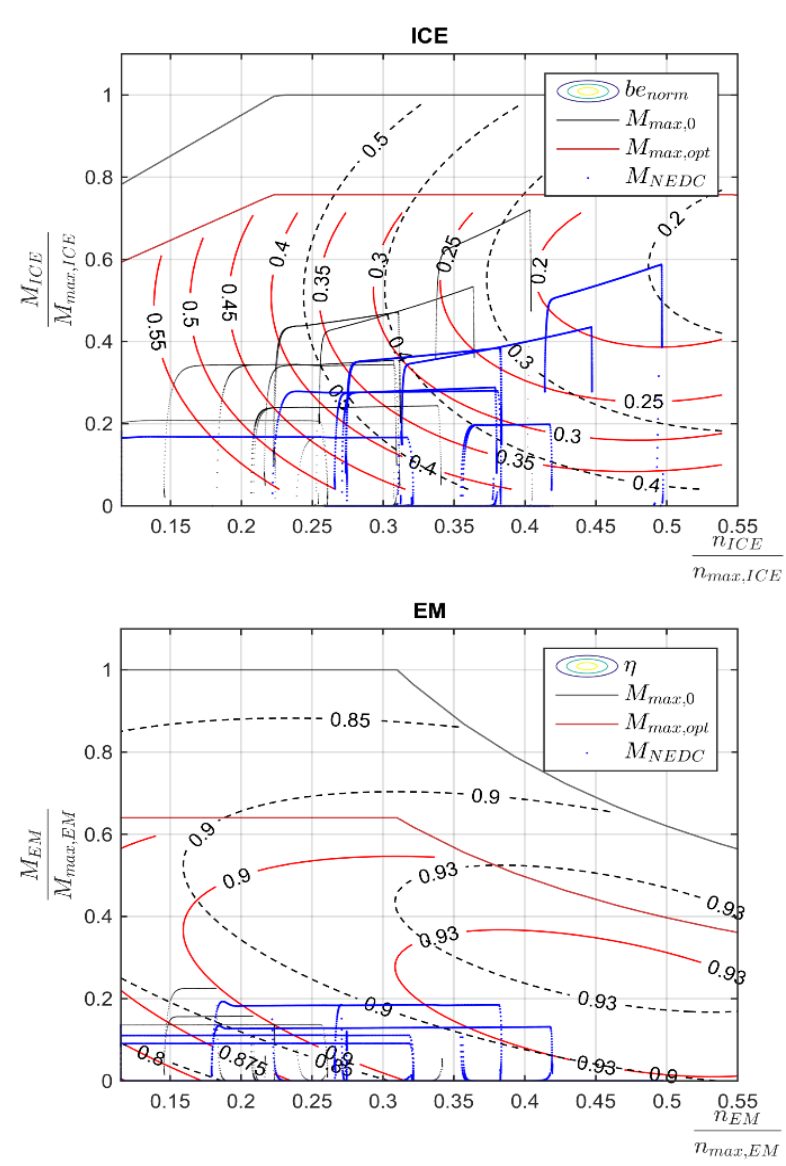

Fig. 11 Results for PHEV (discharged) with variable transmission on NEDC. 

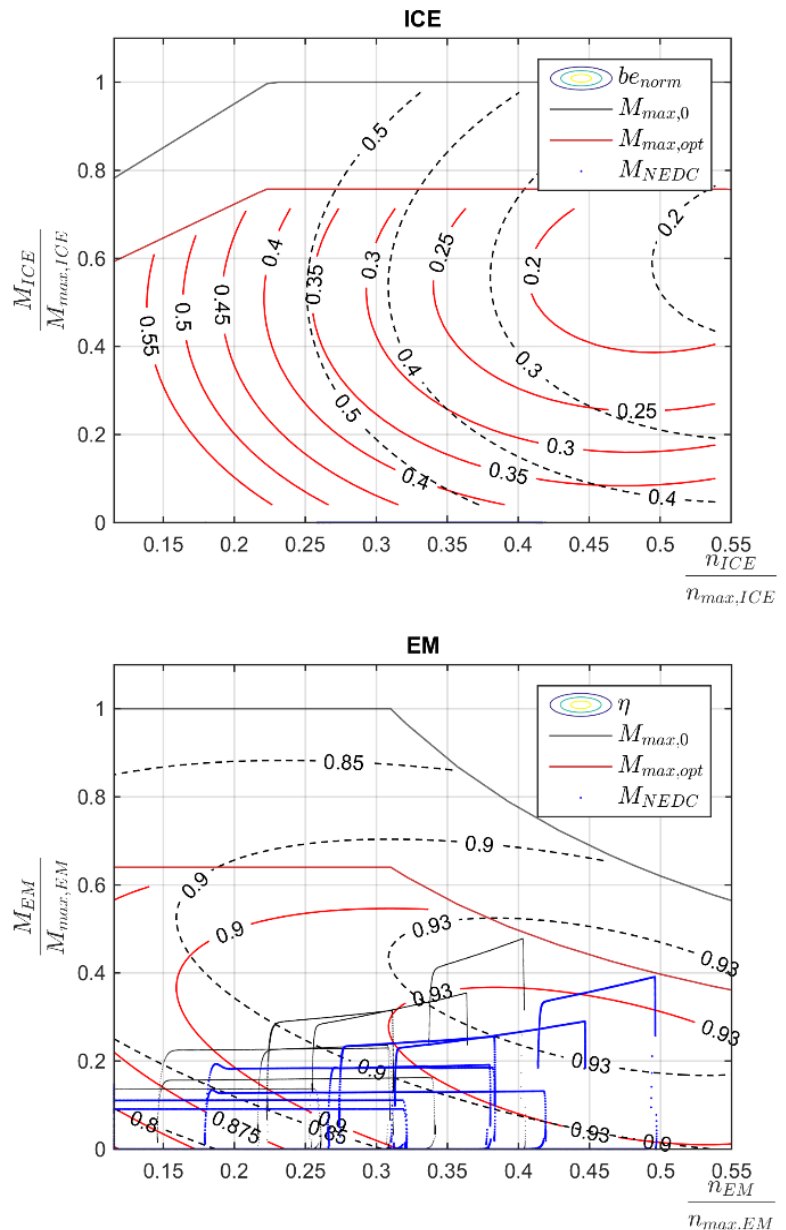

Fig. 12 Results for PHEV (charged) with variable transmission on NEDC.

can be reduced. In this case the reduction of the carbon dioxide emissions is approximately $15 \%$. For the PHEV with charged battery at the beginning of the cycle and variable transmission the results are depicted in Fig. 12. As the cycle is totally driven by using the electrical engine only the maximum torque curve of the ICE is shifted to the minimum value that is needed to follow the speed trajectory with depleted battery. For the EM, the energy demand could be reduced with higher transmission ratios by approximately $13 \%$.

This small sample of taking variable transmission into account shows that integrating more vehicle parameters to the optimization framework has the potential for further energy demand reduction.

\section{Conclusions}

In this paper, a method to determine an optimal propulsion system for two different load cycles has been presented. It has been demonstrated, that the designed method delivers a powertrain that is optimized on the considered load cycle. A BEV design is most beneficial compared to a conventional or parallel hybrid powertrain for the chosen compact car driving the NEDC and WLTC. Although a limited number of powertrain configurations have been investigated, the modular structure of the simulation model is capable to include additional configurations. Furthermore, generic and evolutionary optimization algorithms will be evaluated and additional vehicle parameter will be included in the optimization in future work. The objective in a more general context is to determine an optimal powertrain for different usage profiles. Therefore, user specific load cycles will be considered in conjunction with usage criteria.

\section{References}

[1] German Association of the Automotive Industry. 2015. "Registration of New Vehicles by Country 2014." Statista. Accessed January 5, 2015. http://de.statista.com/statistik /daten/studie/181566/umfrage/neuzulassungen-von-perso nenkraftwagen-nach-laendern/.

[2] Meywerk, M. 2015. Vehicle Dynamics. England: John Wiley \& Sons.

[3] Hoffmann, P. 2010. Hybridvehicles. Vienna: Springer.

[4] Braess, H. H., and Seiffert, U. 2005. Handbook of Automotive Engineering. USA: SAE International.

[5] Reinhardt, R., Hoffamnn, A., and Gerlach, T. 2013. Nonlinear Optimization. Berlin Heidelberg: Springer.

[6] Ulbrich, M., and Ulbrich, S. 2012. Nonlinear Optimization. Basel: Springer.

[7] Nocedal, J., and Wright, S. 2006. Numerical Optimization. New York: Springer.

[8] Federal Environmental Agency. 2014. "Power and Heat Supply." Accessed January 15, 2015. http://www.umweltbundesamt.de/themen/klima-energie/e nergieversorgung/strom-waermeversorgung-in-zahlen. 